\title{
Supratherapeutic anticoagulation at presentation is associated with reduced mortality in nonvariceal upper gastrointestinal hemorrhage
}

Authors

Institutions
James Irwin ${ }^{1,2}$, Reid Ferguson ${ }^{3}$, Frank Weilert ${ }^{1}$, Anthony Smith ${ }^{1}$

${ }^{1}$ Waikato Hospital - Gastroenterology, Hamilton, New Zealand

2 Royal Brisbane and Women's Hospital - Gastroenterology, Brisbane, Queensland, Australia

${ }^{3}$ Auckland University - Medicine, Auckland, New Zealand submitted 23. April 2014

accepted 5. May 2014

\section{Bibliography}

DOI http://dx.doi.org/

10.1055/s-0034-1377287

Published online: 10.7.2014

Endoscopy International Open

2014; 2: E148-E152

(c) Georg Thieme Verlag KG

Stuttgart · New York

E-ISSN 2196-9736

\section{Corresponding author}

\section{James Robert Irwin}

Royal Brisbane and Women's Hospital-Gastroenterology

Butterfield Street

Brisbane, Queensland 4006

Australia

Fax: 0061736368110

jazirwin@gmail.com

James_Irwin@health.qld.gov.au
Introduction: Warfarin is a widely used and easily reversible anticoagulant. Although bleeding is more likely in warfarin users, it may also be more readily treated. This retrospective observational case-control study compares the outcome of acute nonvariceal upper gastrointestinal hemorrhage in warfarin users with a supratherapeutic international normalized ratio (INR) and outcome in non-warfarin users.

Patients and methods: Clinical and endoscopic data for patients presenting with overt upper gastrointestinal hemorrhage were collected between 23rd February 2001 and 12 October 2010.Patients with variceal hemorrhage were excluded. Warfarin users with a supratherapeutic INR ( $\geq$ 3.0) at presentation (supratherapeutic anticoagulation [SA] group) were matched to a cohort with upper gastrointestinal hemorrhage not taking

\section{Introduction}

Patin

Patients who take warfarin are at increased risk of gastrointestinal bleeding [1,2]. Patients taking warfarin typically have significant comorbidity, often related to the indication for which their warfarin was prescribed. Comorbidity is known to be associated with increased mortality in patients with upper gastrointestinal hemorrhage [3]. Previous studies have not adjusted for comorbidity when analysing the outcome of upper gastrointestinal hemorrhage in warfarin users, and therefore they may have overestimated the association between mortality and warfarin use $[4,5]$. The coagulopathy induced by warfarin is readily reversible, providing a treatment strategy that may improve outcome. This effect is likely to be most apparent in patients taking warfarin who have a supratherapeutic international normalized ratio (INR). This analysis compares 30-day mortality from upper gastrointestinal hemorrhage in warfarin users with a supratherapeutic INR and 30-day mortality in non - warfarin users, adjust- warfarin at presentation (control group). Patients were matched by age, sex, Rockall score, year of endoscopy, inpatient or outpatient status, and the presence of disseminated cancer at presentation. The incidence rates of major outcomes in the two groups were compared.

Results: A total of 128 patients (SA group) were matched to 135 control patients. The SA group patients were less likely to die within 30 days ( $6.25 \%$ vs. $15.5 \%$, odds ratio $=0.36, P=0.028$ by Test for Equality of Proportions). There was a trend toward more surgery in the control group ( $5 \%$ vs. $2 \%$ ), and rates of blood transfusion $(77 \%$ vs. $70 \%$ ) were similar in the two groups.

Conclusion: In patients presenting with nonvariceal upper gastrointestinal hemorrhage, a supratherapeutic INR at presentation due to warfarin use is associated with reduced mortality.

ing for potential confounding by matching to a control group for variables known to influence survival in upper gastrointestinal hemorrhage.

\section{Methods \\ $\nabla$}

\section{Patient selection}

The study was conducted in a single regional hospital (Waikato Hospital, Hamilton, New Zealand) between 23rd February 2001 and 12 October 2010. For all patients with upper gastrointestinal hemorrhage at presentation to the hospital or during an admission for a different condition, the following were recorded prospectively in a dedicated upper gastrointestinal bleed database: presenting features, endoscopic diagnosis, presence of stigmata of recent hemorrhage (including Forrest classification of peptic ulceration) [6,7] and administered treatment. Those patients with evidence of variceal bleeding were excluded from the analysis. When a patient had more than one episode of upper gas- 
trointestinal hemorrhage during the study period, only the first event was included.

Additional clinical and laboratory data at presentation were retrospectively collected through review of the clinical record. These data were recorded by an investigator blinded to the endoscopic findings. Data regarding the use of Prothrombinex (pooled purified human coagulation factors II, IX, and X; CSL Ltd, Broadmeadows, Australia), fresh frozen plasma, and platelet and packed red cell transfusion were obtained from a national transfusion database.

All patients taking warfarin who had an INR of 3 or higher were included in a supratherapeutic anticoagulation (SA) group.The optmatch package in $\mathrm{R}[8]$ was used to match these patients to a control group of patients taken from the upper gastrointestinal hemorrhage database who were not taking warfarin at presentation. Patients were matched for age, sex, pre-endoscopy Rockall score, year of upper gastrointestinal hemorrhage, inpatient or outpatient status, and the presence of disseminated cancer at presentation. The year of upper gastrointestinal hemorrhage was analysed as a dichotomous variable, with patients recorded as having had an episode of bleeding in either the first or the second half of the study period. Patients were matched for year of upper gastrointestinal hemorrhage to ensure that changes in referral patterns or in clinical management over the study period did not contribute bias to the outcome. Patients were not well matched on age and presence of disseminated cancer at presentation if the Rockall score alone was used as a representative matching variable. Age and presence of disseminated cancer were therefore included as matching variables. We elected to control confounding by using the Rockall score, and not the Glasgow- Blatchford score, although both are validated as predictors of death in upper gastrointestinal hemorrhage [9].

Patients were considered to have had an upper gastrointestinal hemorrhage if they had hematemesis or coffee ground vomitus, or if they passed melena per rectum. A rebleeding event was defined as further fresh hematemesis, melena with associated hemodynamic instability (defined as pulse rate $>100$ beats/min or systolic blood pressure $<100 \mathrm{mmHg}$ ), or a drop in the hemoglobin level of 20 points or greater and hemodynamic instability subsequent to endoscopy.

\section{Statistics}

The R statistical programming environment [8] was used for statistical analysis. Demographics, baseline characteristics, endoscopic findings, and treatment administered were compared between the two groups to assess the adequacy of matching and potentially confounding factors. Student's $t$ test was used to compare continuous variables, and a chi-square test was used to compare categorical variables. A $P$ value of 0.05 or less was considered significant with the use of two-tailed testing. Mortality was analysed as the primary outcome. The need for surgery, occurrence of rebleeding, and need for transfusion were analysed as secondary outcomes.

\section{Results}

$\nabla$

A total of 1603 patients met the inclusion criteria, 128 of whom were taking warfarin with an INR at admission of 3 or higher (the SA group). They were matched to 135 patients not taking warfarin (the control group). The baseline characteristics, with the clinical and laboratory parameters at presentation, are listed in $\bullet$ Ta- ble 1 for the two groups and compared with those of all patients in the upper gastrointestinal hemorrhage database. The rate of comorbidity in the SA and control groups was significantly higher than in the unselected upper gastrointestinal hemorrhage cohort. In the SA group, 88 patients were receiving warfarin as stroke prophylaxis because of atrial fibrillation, 17 for a prosthetic heart valve, 16 for a prosthetic heart valve and atrial fibrillation, 4 for venous thromboembolism, and 3 for other reasons. Findings at endoscopy are listed in $\bullet$ Table 2 . There was a trend toward more benign endoscopic diagnoses in the SA group.In the patients with peptic ulceration, there was no significant difference in Forrest classification between the two groups (SA group: 10 of 42 class I, 5 of 42 class IIa, 7 of 42 class IIb; control group: 13 of 56 class I, 9 of 56 class IIa, 4 of 56 class IIb). There was no significant difference in endoscopic therapy administered (৫ Table 3).

Reversal of coagulopathy is outlined in $\bullet$ Table 4. Normalization of coagulopathy (INR < 1.5) was achieved in 93 of 128 patients, 79 within 48 hours and 59 within 24 hours of presentation. Warfarin was restarted in 61 of 128 patients at a median of 7.6 days (interquartile range 3.4-31 days) after presentation. Intravenous heparin was used in 17 patients for a median duration of 2.2 days (interquartile range 2.2-4.0 days) after presentation.

The 30-day mortality, surgery, and rebleeding rates are listed in - Table 5. The control group had a higher number of deaths due to myocardial infarction and general decline ( $\bullet$ Table 6 ). Two patients in each group died of uncontrolled bleeding. The excess mortality observed in the control group occurred in the first 10 days following presentation ( $\bullet$ Fig. 1 ).

\section{Discussion \\ $\nabla$}

This retrospective, observational case-control analysis demonstrates that a supratherapeutic INR at presentation is associated with a reduced mortality rate among patients presenting with upper gastrointestinal hemorrhage when control for comorbidity is implemented. Patients were well matched for the variables associated with mortality in upper gastrointestinal hemorrhage $[3,11]$, although there was a trend toward higher rates of comorbidity, higher blood urea levels, and lower hemoglobin concentrations in the SA group.

The 30 -day mortality rate of $15.5 \%$ in the control group was significantly higher than most published figures for upper gastrointestinal hemorrhage, and higher than the overall rate of $9.2 \%$ for the patients in our study [12-15]. This is a reflection of the degree of comorbidity in the control group of patients, in whom the mean pre-endoscopy Rockall score was 3.89. This is comparable to the mortality rate observed in the original Rockall cohort (pre-endoscopy Rockall scores of 3 and 4, 30-day mortality rates of $11 \%$ and $24.6 \%$, respectively) [3].

Anticoagulation was reversed at presentation in the majority of SA patients, and an INR of less than 1.5 was achieved within 24 hours in 59 of 128 patients. Anticoagulation was withheld for 30 days or longer in the majority of patients; for some, however, the risk of thromboembolism was perceived to be high, and warfarin or intravenous heparin was commenced within this period.

A possible explanation for the observed difference in mortality is that supratherapeutic anticoagulation produces clinically significant bleeding from lower-risk mucosal lesions. Patients can be readily treated by reversal of anticoagulation in addition to standard management (endoscopic therapy and intravenous in- 


\begin{tabular}{|c|c|c|c|c|c|}
\hline & $\begin{array}{l}\text { SA } \\
(n=128)\end{array}$ & $\begin{array}{l}\text { Control } \\
(n=135)\end{array}$ & $P$ value & $\begin{array}{l}\text { Unmatched } \\
(n=1475)\end{array}$ & $\begin{array}{l}P \text { value } \\
\text { (SA vs. unmatched) }\end{array}$ \\
\hline \multicolumn{6}{|l|}{ Continuous variables } \\
\hline Age, $y$ & 72.04 & 72.14 & 0.95 & 68.43 & $<0.001^{2}$ \\
\hline ASA score [10] & 2.72 & 2.49 & $0.01^{1}$ & 2.45 & $<0.001^{2}$ \\
\hline Rockall score & 3.92 & 3.87 & 0.80 & 3.16 & $<0.001^{2}$ \\
\hline $\mathrm{SBP}, \mathrm{mmHg}$ & 118.05 & 119.21 & 0.73 & 125.87 & $<0.001^{2}$ \\
\hline Pulse rate, beats/min & 86.38 & 88.21 & 0.48 & 88.36 & 0.32 \\
\hline Hemoglobin, $\mathrm{g} / \mathrm{L}$ & 89.95 & 96.65 & 0.07 & 101.20 & $<0.001^{2}$ \\
\hline Platelets, $\times 10^{9} / \mathrm{L}$ & 257.05 & 274.02 & 0.28 & 279.67 & $0.03^{1}$ \\
\hline Creatinine, $\mu \mathrm{mol} / \mathrm{L}$ & 130.45 & 119.87 & 0.30 & 126.41 & 0.65 \\
\hline Urea, mmol/L & 20.60 & 15.54 & $<0.001^{2}$ & 14.73 & $<0.001^{2}$ \\
\hline \multicolumn{6}{|l|}{ Categorical variables } \\
\hline Inpatient & 0.16 & 0.11 & 0.37 & 0.24 & $0.05^{1}$ \\
\hline Male sex & 0.67 & 0.64 & 0.64 & 0.59 & 0.10 \\
\hline Second half of study & 0.62 & 0.59 & 0.68 & 0.48 & $<0.001^{2}$ \\
\hline Fresh hematemesis & 0.20 & 0.30 & 0.08 & 0.28 & $0.05^{1}$ \\
\hline Disseminated cancer & 0.02 & 0.02 & 1.00 & 0.04 & 0.32 \\
\hline Ischemic heart disease & 0.41 & 0.37 & 0.55 & 0.24 & $<0.001^{2}$ \\
\hline Cardiac failure & 0.27 & 0.19 & 0.16 & 0.12 & $<0.001^{2}$ \\
\hline Stroke & 0.17 & 0.07 & $0.03^{1}$ & 0.11 & $0.03^{1}$ \\
\hline Renal failure & 0.11 & 0.10 & 0.88 & 0.10 & 0.88 \\
\hline COPD & 0.16 & 0.12 & 0.38 & 0.09 & $0.01^{1}$ \\
\hline Diabetes & 0.28 & 0.21 & 0.21 & 0.19 & $0.02^{1}$ \\
\hline Rheumatoid arthritis & 0.01 & 0.00 & 0.98 & 0.01 & 0.89 \\
\hline Aspirin & 0.47 & 0.59 & 0.06 & 0.46 & 0.88 \\
\hline NSAID & 0.08 & 0.20 & $0.01^{1}$ & 0.19 & $<0.001^{2}$ \\
\hline PPI & 0.23 & 0.26 & 0.75 & 0.26 & 0.64 \\
\hline
\end{tabular}

SA, supratherapeutic anticoagulation; unmatched, unmatched cohort not taking warfarin at presentation; ASA, American Society of Anesthesiologists; SBP, systolic blood pressure; COPD, chronic obstructive pulmonary disease; NSAID, nonsteroidal anti-inflammatory drug; PPI, proton pump inhibitor.

The $P$ value represents the significance of two-tailed Student's $t$ test for the difference between two means for continuous variables, and the significance of the difference of proportions for categorical variables.

$1 P<0.05$.

$2 P<0.001$.

\begin{tabular}{|llllll|}
\hline & Control $(\mathbf{n = 1 3 5 )}$ & Proportion & SA $(\mathbf{n = 1 2 8})$ & Proportion & $P$ value \\
\hline Gastric ulcer & 34 & 0.25 & 20 & 0.16 & 0.08 \\
\hline Duodenal ulcer & 24 & 0.18 & 25 & 0.20 & 0.84 \\
\hline Vascular lesion & 9 & 0.07 & 7 & 0.05 & 0.88 \\
\hline Esophagitis & 18 & 0.13 & 15 & 0.12 & 0.83 \\
\hline Esophageal cancer & 1 & 0.01 & 1 & 0.01 & 1.00 \\
\hline Gastric cancer & 7 & 0.05 & 3 & 0.02 & 0.38 \\
\hline Mallory-Weiss tear & 5 & 0.04 & 4 & 0.03 & 1.00 \\
\hline Gastric erosion & 16 & 0.12 & 23 & 0.18 & 0.22 \\
\hline Duodenal erosion & 6 & 0.04 & 11 & 0.09 & 0.26 \\
\hline Normal endoscopy & 22 & 0.16 & 24 & 0.19 & 0.72 \\
\hline
\end{tabular}

Table 1 Baseline characteristics and clinical parameters at presentation.

SA, supratherapeutic anticoagulation.

The $P$ value represents the significance of difference of proportions. $P<0.05$ is considered significant.

Table 2 Endoscopic diagnosis.

\begin{tabular}{|lllll|}
\hline & $\begin{array}{l}\text { Control } \\
(\mathbf{n = 1 3 5 )}\end{array}$ & Proportion & SA & $\begin{array}{l}\text { Proportion } \\
(\mathbf{n = 1 2 8 )}\end{array}$ \\
\hline Intravenous PPI & 47 & 0.31 & 38 & 0.30 \\
\hline Adrenaline injection & 31 & 0.20 & 23 & 0.18 \\
\hline Diathermy coagulation & 20 & 0.13 & 16 & 0.12 \\
\hline Hemostatic clip placement & 11 & 0.07 & 12 & 0.09 \\
\hline
\end{tabular}

Table 3 Therapy administered.

SA, supratherapeutic anticoagulation; PPI, proton pump inhibitor.

fusion of omeprazole). A higher proportion of patients in the SA group with only gastric or duodenal erosions seen at endoscopy supports this hypothesis. A trend toward an increased rate of surgery in the control group is also supportive. However, rates of rebleeding were not different between the two groups.
There were notably fewer cardiac events in the SA group. The small number of deaths in this study precludes statistical analysis of the causes of death in a meaningful way. It is biologically plausible that there may be a beneficial effect of anticoagulation on the cardiac circulation during a time of physiologic stress, such 
Table 4 Treatment administered to reverse anticoagulation.

\begin{tabular}{|lcl|} 
& SA $(\mathbf{n = 1 2 8})$ & Proportion \\
\hline No reversal of anticoagulation & 6 & 0.05 \\
\hline Vitamin K & 107 & 0.84 \\
\hline FFP & 68 & 0.53 \\
\hline Prothrombinex & 6 & 0.05 \\
\hline FFP and Prothrombinex & 22 & 0.17 \\
\hline
\end{tabular}

SA, supratherapeutic anticoagulation; FFP, fresh frozen plasma.

Table 5 Outcome variables.

\begin{tabular}{|lccl|} 
& Control $(\mathbf{n = 1 3 5 )}$ & SA (n=128) & P value \\
\hline Death within 30 days & 21 & 8 & $0.028^{1}$ \\
\hline Surgery & 7 & 2 & 0.202 \\
\hline Rebleed & 10 & 7 & 0.698 \\
\hline Transfusion & 95 & 98 & 0.32
\end{tabular}

The $\mathrm{P}$ value represents the significance of the difference of proportions. $1 P<0.05$.

Table 6 Cause of death

\begin{tabular}{|lll|}
\hline & Control Group & SA \\
\hline Uncontrolled gastrointestinal bleeding & 2 & 2 \\
\hline Myocardial infarction & 8 & 2 \\
\hline General deterioration & 5 & 1 \\
\hline Multiple organ failure & 1 & 1 \\
\hline Pneumonia & 3 & 0 \\
\hline Respiratory failure due to fluid overload & 1 & 0 \\
\hline Aspiration and respiratory arrest & 1 & 0 \\
\hline Bradycardia, hypotension & 0 & 1 \\
\hline Embolic stroke & 0 & 1 \\
\hline
\end{tabular}

as during upper gastrointestinal hemorrhage. Sung and colleagues demonstrated that stopping antiplatelet therapy with aspirin during admission for acute upper gastrointestinal hemorrhage was associated with increased mortality, and they postulated that this was due to a loss of the cardioprotective effect of aspirin [16]. Reduction in myocardial infarction due to warfarin-induced anticoagulation could be an alternative explanation for the observed difference in mortality.

Factor X inhibitors (rivaroxaban, apixaban) and direct thrombin inhibitors (dabigatran) have been shown to be effective in the prophylaxis of stroke in atrial fibrillation, and in the prophylaxis of venous thromboembolism [17-22]. Is the lower mortality in upper gastrointestinal hemorrhage associated with warfarin use that we have observed likely to translate to patients taking these medications? A major concern with factor X inhibitors and direct thrombin inhibitors is that in the event of acute hemorrhage, they are not readily reversible. Current therapeutic options in the setting of life-threatening bleeding include hemodialysis to remove the drug (dabigatran), the administration of Prothrombinex (rivaroxiban, apixaban), or the administration of activated factor VIIa (all). There is little published experience of the clinical efficacy of these strategies, and the normalization of anticoagulation for factor $\mathrm{X}$ and direct thrombin inhibitors may depend on unassisted metabolism of the drug. Serum half-lives vary from 10 to 15 hours $[17,19,23]$, a delay that may be significant in the context of an acute bleed.

Published randomized trials comparing factor $\mathrm{X}$ and direct thrombin inhibitors with warfarin did not specify a rate of mortality due to gastrointestinal bleeding [16-21]. Rates of major

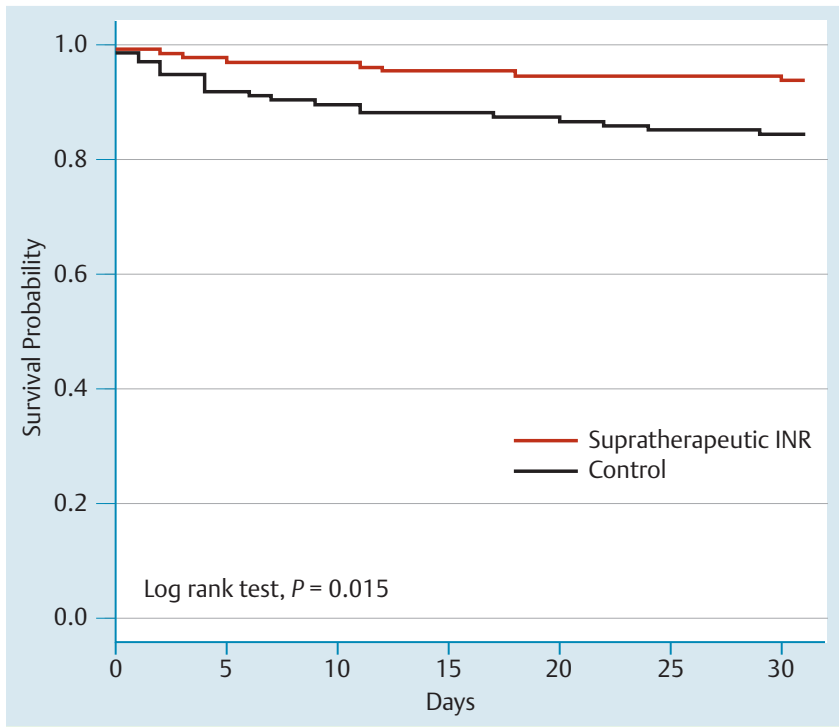

Fig. 1 Survival difference - Kaplan-Meier plot.

gastrointestinal bleeding were higher for all of these agents when compared with warfarin; however, mortality from bleeding was lower primarily because of a reduced rate of fatal intracranial hemorrhage. As clinical experience accumulates for upper gastrointestinal hemorrhage associated with the use of factor $\mathrm{X}$ inhibitors or direct thrombin inhibitors, the outcome of these patients will become clearer.

Because it is retrospective in nature, this analysis is subject to potential sources of bias. The most important of these is that the inclusion of patients in the study was subject to the performance of endoscopy, and the threshold for performing endoscopy may have differed between patients taking warfarin and those not taking warfarin. However, the proportion of patients receiving blood transfusion, and the clinical and laboratory parameters suggestive of heavy bleeding at presentation (serum urea and hemoglobin levels, pulse rate, and blood pressure), were similar between the SA patients and those in the control group, suggesting that the severity of bleeding was similar in the two groups.

\section{Summary}

Despite high levels of comorbidity, which are known to predict a poor outcome, a supratherapeutic INR at presentation due to warfarin use was associated with reduced mortality in patients with nonvariceal upper gastrointestinal hemorrhage. Reduced mortality may have been due to the effect of warfarin anticoagulation inducing bleeding from lesser mucosal lesions, with such bleeding more effectively controlled by reversal of anticoagulation in addition to endoscopic therapy and proton pump inhibitor infusion. Alternatively, the lower mortality rate may have been due to a reduced incidence of myocardial infarction during episodes of bleeding as a result of the anticoagulant effect of warfarin.

Competing interests: None. 


\section{References}

1 EAFT (European Atrial Fibrillation Trial) Study Group. Secondary prevention in non-rheumatic atrial fibrillation after transient ischaemic attack or minor stroke. Lancet 1993; 342: 1255-1262

2 Warfarin versus aspirin for prevention of thromboembolism in atrial fibrillation: Stroke Prevention in Atrial Fibrillation II Study. Lancet 1994; 343: 687-691

3 Rockall TA, Logan RF, Devlin HB et al. Risk assessment after acute upper gastrointestinal haemorrhage. Gut 1996; 38: 316-321

4 Thomopoulos KC, Mimidis KP, Theocharis GJ et al. Acute upper gastrointestinal bleeding in patients on long-term oral anticoagulation therapy: endoscopic findings, clinical management and outcome. World J Gastroenterol 2005; 11: $1365-1368$

5 Choudari CP, Rajgopal C, Palmer KR. Acute gastrointestinal haemorrhage in anticoagulated patients: diagnoses and response to endoscopic treatment. Gut 1994; 35: 464-466

6 Forrest JA, Finlayson ND, Shearman DJ. Endoscopy in gastrointestinal bleeding. Lancet 1974; 2: 394-397

7 Laine L, Peterson WL. Bleeding peptic ulcer. N Engl J Med 1994; 331: 717-727

8 The R Core Team. R: a language and environment for statistical computing. Vienna, Austria: The R Project for Statistical Computing; 2011: http://www.R-project.org Accessed May 9, 2014

9 Stanley AJ, Dalton HR, Blatchford $O$ et al. Multicentre comparison of the Glasgow Blatchford and Rockall Scores in the prediction of clinical endpoints after upper gastrointestinal haemorrhage. Aliment Pharmacol Ther 2011; 34: 470-475

10 Owens WD, Felts JA, Spitznagel EL Jr. ASA physical status classifications: a study of consistency of ratings. Anesthesiology 1978; 49: 239-243

11 Marmo R, Koch M, Cipolletta $L$ et al. Predictive factors of mortality from nonvariceal upper gastrointestinal hemorrhage: a multicenter study. Am J Gastroenterol 2008; 103: 1639-1647; quiz 1648

12 Rockall TA, Logan RF, Devlin HB et al. Incidence of and mortality from acute upper gastrointestinal haemorrhage in the United Kingdom.
Steering Committee and members of the National Audit of Acute Upper Gastrointestinal Haemorrhage. BMJ 1995; 311: 222 - 226

13 Blatchford O, Davidson LA, Murray WR et al. Acute upper gastrointestinal haemorrhage in west of Scotland: case ascertainment study. BMJ 1997; 315: $510-514$

14 Lewis JD, Bilker WB, Brensinger $C$ et al. Hospitalization and mortality rates from peptic ulcer disease and GI bleeding in the 1990s: relationship to sales of nonsteroidal anti-inflammatory drugs and acid suppression medications. Am J Gastroenterol 2002; 97: 2540 - 2549

15 Loperfido S, Baldo V, Piovesana $E$ et al. Changing trends in acute upperGI bleeding: a population-based study. Gastrointest Endosc 2009; 70: $212-224$

16 Sung JJY, Lau JYW, Ching JYL et al. Continuation of low-dose aspirin therapy in peptic ulcer bleeding: a randomized trial. Ann Intern Med 2010; 152: $1-9$

17 Connolly SJ, Ezekowitz MD, Yusuf S et al. Dabigatran versus warfarin in patients with atrial fibrillation. N Engl J Med 2009; 361: 1139-1151

18 Patel MR, Mahaffey KW, Garg J et al. Rivaroxaban versus warfarin in nonvalvular atrial fibrillation. N Engl J Med 2011; 365: 883 - 891

19 Granger CB, Alexander JH, McMurray JJV et al. Apixaban versus warfarin in patients with atrial fibrillation. N Engl J Med 2011; 365: 981 -992

20 Schulman S, Kearon C, Kakkar AK et al. Extended use of dabigatran, warfarin, or placebo in venous thromboembolism. N Engl J Med 2013; 368: 709-718

21 Büller HR, Prins $M H$, Lensin AW. EINSTEIN-PE Investigators. et al. Oral rivaroxaban for the treatment of symptomatic pulmonary embolism. N Engl J Med 2012; 366: 1287-1297

22 Agnelli G, Buller HR, Cohen A et al. Oral apixaban for the treatment of acute venous thromboembolism. N Engl J Med 2013; 369: 799-808

23 Weinz C, Schwarz T, Kubitza D et al. Metabolism and excretion of rivaroxaban, an oral, direct factor Xa inhibitor, in rats, dogs, and humans. Drug Metab Dispos 2009; 37: 1056-1064 